\title{
COMENTÁRIO EDITORIAL CUIDADOS A TOMAR NOS ARTIGOS COM PESQUISA QUALITATIVA
}

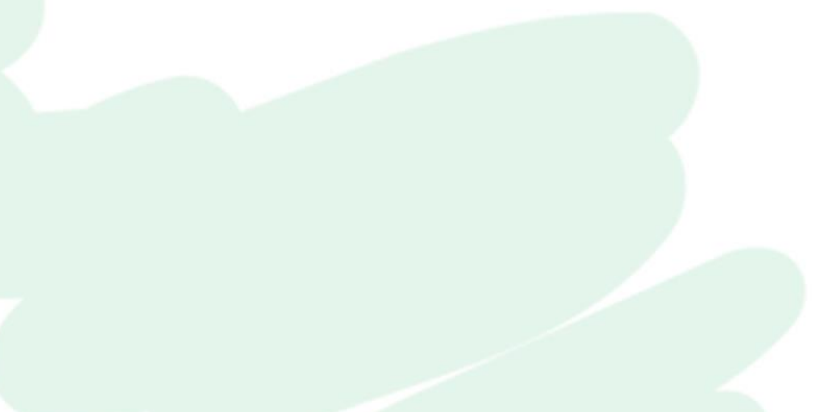

Not everything that van be counted counts, and not everything that counts can be counted.
Fernando Antonio Ribeiro Serra Editor Científico RIAE

Universidade Nove de Julho - UNINOVE

Programa de Pós-Graduação em Administração

\section{Manuel Aníbal Silva Portugal Vasconcelos Ferreira} Editor Adjunto RIAE

Universidade Nove de Julho - UNINOVE Programa de Pós-Graduação em Administração
Na RIAE/IJSM recebemos para avaliação, e publicamos, uma quantidade substancial de artigos que utilizam métodos de pesquisa qualitativa. Nos últimos três anos - de 2014 a 2016 -, não considerando as seções de perspectivas, artigos tecnológicos e de resenhas, quase $65 \%$ dos artigos publicados na RIAE podem ser classificados como seguindo abordagens qualitativas. Isto praticamente o dobro número de artigos baseados em métodos quantitativos publicados no mesmo período.

Como editores e pesquisadores, não temos, pessoalmente, uma preferência absoluta por qualquer metodologia em específico, seja esta mais assente em métodos quantitativos (entenda-se que por estudos quantitativos significamos aqueles com tratamentos estatísticos) ou qualitativos, ou mesmo por artigos conceituais ou teóricos. No entanto, há evidências que os pesquisadores mostram-se mais confortáveis com determinados métodos (Aguinis et al., 2009), e que persistem no uso de determinados métodos (Podsakoff \& Dalton, 1987). No Brasil, a diferença, em contraste com o que vemos nos principais periódicos internacionais da área e nos principais eventos como os encontros da Academy of Management (AoM) ou Academy of International Business (AIB), é a predominância do uso de métodos qualitativos (Serra, Fiates \& Ferreira, 2008; Barbosa, Ponte, Oliveira \& Moura, 2008; Nascimento, Junqueira \& Martins, 2010). A relativa prevalência de métodos qualitativos poderia indiciar maior competência, mas nas submissões à RIAE/ISJM detectamos, como confirmam os pareceres dos revisores, muitas deficiências, e talvez inclusive mais significativas que nos artigos submetidos com métodos quantitativos.

A preocupação com a maior qualidade dos estudos qualitativos não é exclusivamente brasileira, e diversos editoriais de periódicos - onde a maioria dos artigos publicados são quantitativos - incluem recomendações para a melhor elaboração e para maior possibilidade de aprovação (ver, por exemplo, Bansal \& Corley, 2012; Reay, 2014; Bettis et al., 2015). Talvez pelas deficiências na sua elaboração (Fiates, 
Serra \& Martins, 2014), que existe uma predominância de artigos quantitativos internacionalmente (Phelan, Ferreira \& Salvador, 2002; Serra, Fiates \& Ferreira, 2008). Face a nossa maior propensão em fazer pesquisas qualitativas no Brasil, as preocupações com a qualidade exigem atenção e talvez sinalizem que a realização de estudos qualitativos exige preparação e conhecimento que raramente é provido nos programas stricto sensu. Ou seja, ensinar os estudantes que não basta fazer umas entrevistas ou escolher casos de conveniência, ensinar sobre técnicas de condução das pesquisas e técnicas de análise dos dados, entre tantos aspectos fundacionais a pesquisa de qualidade.

Neste comentário editorial, pretendemos contribuir para aumentar a qualidade, ou, pelo menos o entendimento, dos estudos qualitativos para aumentar a possibilidade de publicação (Falaster et al., 2016). Especificamente, discutimos de forma sintética, que não dispensa o estudo, as características dos artigos (e estudos) qualitativos e sugerimos alguns cuidados para a preparação dos artigos para submissão. Este comentário editorial, mais do que um roteiro a seguir, tem como base a nossa experiência como editores, as recomendações que fazemos nas revisões, ou que os revisores fazem nos trabalhos que avaliam, e, também, as contribuições de outros editoriais e artigos sobre pesquisa qualitativa. Parece-nos óbvio que as pretensões de publicação internacional requerem uma ação conducente a realização de pesquisa na qualidade necessária a essa publicação.

\section{CARACTERÍSTICAS DA PESQUISA QUALITATIVA}

Como qualquer pesquisa, um artigo qualitativo precisa considerar aspectos como (Kohler, 2016): clareza, propósito e transparência. A clareza relacionase com uma redação clara, com informação relevante e de forma que os leitores possam entender. Cada parágrafo precisa expressar uma ideia e se conectar adequadamente com os parágrafos seguintes (Ferreira, 2015). Apresentar o propósito, significa deixar clara a motivação e objetivo para o trabalho. É comum designarmos por questão de pesquisa o foco que orienta todo o trabalho (Kohler, 2016). A transparência implica em prover informações relevantes de como a pesquisa foi conduzida, os percalços e ajustes no caminho. Está relacionada com o rigor na realização da pesquisa nos estudos qualitativos (Gibbert \& Ruigrok, 2010).

Num editorial do Academy of Management Journal (AMJ), Bansal e Corley (2012) apresentaram as características dos artigos qualitativos, em contraponto com os artigos quantitativos, a partir de respostas de revisores. Sintetizamos, na tabela 1, uma comparação entre os estudos quantitativos e os qualitativos. A comparação já permite antecipar algumas diferenças que mostram aspectos a cuidar no planejamento e elaboração de estudos, e artigos, qualitativos. Além dos aspetos constantes da tabela, merece uma referência nossa a forma. As deficiências na forma são frequentes e recomendamos em nossas revisões que os artigos sigam uma estrutura comum e definida, pois facilita a compreensão pelos leitores e revisores (Ferreira, 2015). Assim, e embora possa haver nos artigos qualitativos um pouco mais de flexibilidade, em virtude da teoria emergir dos dados e da discussão em relação à revisão de literatura interagir com os dados (Bansal \& Corley, 2012), recomendamos fortemente aos autores que busquem separar os resultados da discussão. Na discussão voltamos a teoria e não aos resultados em si mesmos. Acreditamos que facilite a compreensão do leitor, mas, sobretudo, evita que o artigo fique somente na discussão sobre os resultados, ou que fique meramente na concordância e discordância em relação à literatura anterior, não evidenciando a contribuição. 
Tabela 1 - Características das pesquisas quantitativas e qualitativas

\begin{tabular}{|c|c|c|c|}
\hline & Pesquisa Quantitativa & Pesquisa Qualitativa & Consequência para a pesquisa qualitativa \\
\hline Organização & $\begin{array}{c}\text { Introdução; Revisão de Literatura; } \\
\text { Modelo Conceitual e Hipóteses; Método; } \\
\text { Resultados; Discussão; Conclusão }\end{array}$ & $\begin{array}{l}\text { Introdução; Revisão de Literatura } \\
\text { (encaminha a lacuna); Método; } \\
\text { Resultados; Discussão (contribuição } \\
\text { emerge da interconexão) }\end{array}$ & \begin{tabular}{|c|} 
Na quanti existe um plano rigorosamente \\
executado desde o início. Na quali existe \\
exploração de ideias e adaptação. Na quali é um \\
processo, não um plano. \\
\end{tabular} \\
\hline $\begin{array}{c}\text { Contrução e teste de } \\
\text { teorias }\end{array}$ & Teste de hipóteses normalmente & Pesquisa indutiva normalmente & $\begin{array}{l}\text { Precisa construir o contexto para a pergunta de } \\
\text { pequisa. Mais complicado estabelecer a lacuna } \\
\text { teórica e o caso. }\end{array}$ \\
\hline $\begin{array}{l}\text { Parte inicial do trabalho } \\
\text { (introdução e revisão da } \\
\text { literatura) }\end{array}$ & $\begin{array}{c}\text { A teoria (modelo conceitual) é } \\
\text { apresentado a partir do desenvolvimento } \\
\text { das hipóteses. Dimensão maior que no } \\
\text { qualitativo. }\end{array}$ & $\begin{array}{l}\text { Ela é curta e multipropósto. A teoria } \\
\text { emerge dos dados. }\end{array}$ & \begin{tabular}{|l|} 
Pergunta de pesquisa normalmente "como" e \\
"porque". Muitas vezs as duas. Esta parte tem o \\
objetivo de fisgar o leitor. Apresenta a lacuna na \\
conversação teórica atual. Abre caminho para os \\
dados textuais.
\end{tabular} \\
\hline Método & $\begin{array}{c}\text { Bem estruturado, com base em dados } \\
\text { bem conhecidos e especificados, e testes } \\
\text { estatísticos. }\end{array}$ & $\begin{array}{l}\text { Não linear, conforme o caminho, com } \\
\text { amplitude significativa nas } \\
\text { entrevistas/etnografias e técnicas de } \\
\text { análise. }\end{array}$ & $\begin{array}{l}\text { Necessidade de explicar detalhadamente as fontes } \\
\text { de dados e análise. A explicação deve ser } \\
\text { coerente e convincente. Seção maior e mais } \\
\text { complexa que nos quantitativos. }\end{array}$ \\
\hline $\begin{array}{l}\text { Parte final do trabalho } \\
\text { (resultados e discussão) }\end{array}$ & $\begin{array}{c}\text { Apresentação estruturada dos resultados } \\
\text { empíricos e discussão com as implicações } \\
\text { para a teoria e para a prática. }\end{array}$ & $\begin{array}{c}\text { Além do que foi colocado para quanti, } \\
\text { integrar os dados e teoria de forma que se } \\
\text { conectem, apresente a teoria emergente e } \\
\text { adiscussão com a literatura. }\end{array}$ & $\begin{array}{l}\text { É mais complexo pela interdependência entre } \\
\text { teoria, análise de dados e contribuição téorica. } \\
\text { Difícil de sintetizar e reduzir os dados em tabelas. } \\
\text { É preciso mostrar os dados e não só decrever. }\end{array}$ \\
\hline
\end{tabular}

Fonte: Adaptado de Bansal e Corley (2012). 
Cuidados a Tomar nos Artigos com Pesquisa Qualitativa

Olhando a tabela, fica evidente que a essência do trabalho qualitativo e quantitativo é similar na busca por responder a uma questão de pesquisa. A estrutura do artigo não resulta da metodologia, mas sim das etapas do processo de pesquisa; e o processo é comum independentemente da metodologia usada. Por exemplo, os artigos quantitativos seguem um plano definido desde o seu início, com a modelagem conceitual que suporta as hipóteses sendo formulada pelos autores antes dos elementos metodológicos e do teste das hipóteses, ao que se segue uma discussão (com reforço da contribuição para a teoria e/ou a prática, as limitações e sugestões de pesquisa). Seguir a estrutura convencional facilita a identificação da contribuição e, no geral, simplifica a leitura porque os leitores esperam ver certas componentes em certos locais do artigo. A mesma racionalidade deve ser seguida nos estudos qualitativos, embora possamos admitir que nos artigos qualitativos a pesquisa possa conter um processo de descoberta (Savall et al., 2008; Gioia, Corley \& Hamilton, 2012), sobretudo no momento da escrita dos resultados e da discussão.

Certamente que há diferenças entre os trabalhos quantitativos e qualitativos. No essencial essas diferenças são bem entendidas pelos pesquisadores. Desde logo, um estudo qualitativo terá dados e informações, mas que não são sujeitos a testes estatísticos (por exemplo, de estatística multivariada). Também não têm hipóteses que serão testadas. Mas, podem ter propostas materializadas em proposições derivadas da teoria que orientam o leitor na leitura e interpretação dos dados. Um estudo qualitativo pode visar a construção de teoria, mas também visar o teste da teoria.

É óbvio que muitas deficiências encontradas nos artigos submetidos não são do foro metodológico, mas antes são simplesmente pesquisas mal planejadas e pobremente conduzidas, sem realmente haver um foco teórico e sem haver um objetivo ou questão de pesquisa. Aliás, estas deficiências podem ocorrer, e ocorrem, em todos os tipos de trabalhos. Ainda assim, é relevante entender que os estudos qualitativos podem ter especificidades quando, por exemplo, visam construir e testar teorias por meio de uma construção exploratória e indutiva. Muitos pesquisadores, no entanto, consideram que esta abordagem não atende ao padrão de qualidade necessário para os trabalhos científicos (Gioia, Corley \& Hamilton, 2012). Estruturar a apresentar os trabalhos de forma rigorosa é fundamental para ganhar a confiança dos avaliadores e conseguir uma boa publicação em qualquer tipo de artigo.

\section{AS SETE ESTRATÉGIAS DE REAY}

O editorial de Reay (2014) é uma peça excelente como auxiliar que nos orienta na análise de um artigo qualitativo. A Editora sugere sete estratégias a considerar nos artigos qualitativos. Ao longo da apresentação das estratégias, vamos apresentar alguns exemplos de artigos qualitativos. Usaremos como exemplos artigos de nossa autoria e publicados em outros periódicos, mas vale realçar que o leitor pode encontrar exemplos melhores nos principais periódicos da área. Também vale observar que, apesar do editorial recente sobre pesquisa qualitativa de Bettis et al. (2015) publicado no Strategic Management Journal, não foram publicados no SMJ artigos qualitativos nos últimos anos. Independentemente da tradição do principal periódico em estratégia de publicar artigos quantitativos, acreditamos que os estudos qualitativos sejam importantes para certos fenômenos e conceitos que dependem de processos.

- Estratégia \#1 - garantir a qualidade e quantidade de dados

Reay recomenda ter dados em qualidade e quantidade suficiente. Os estudos qualitativos podem necessitar de maiores explicações para mostrar a qualidade dos dados e formas como forma coletados e tratados. Usualmente, talvez não se utilizem de amostras grandes, mas a quantidade de informação a ser analisada é importante. Ou seja, realizar 5 ou 8 entrevistas superficiais apenas pode não ser suficiente. Questões como a triangulação dos dados, os roteiros, os procedimentos, a própria seleção dos entrevistados, são todos elementos a atender.

Nos estudos qualitativos, em especial os estudos de caso, é necessário o acesso a múltiplas fontes de dados, como arquivos, observações de campo, documentos, mas especialmente entrevistas, normalmente semiestruturadas (Gioia et al., 2012). Ainda assim, estes estudos podem ser baseados em poucas fontes de dados. Por exemplo, no estudo de caso da Gradiente - um estudo longitudinal de 35 anos sobre declínio organizacional -, foram utilizados "1.472 documentos, principalmente secundários, levantados de publicações de mercado, dissertações e artigos... (e)stavam incluídos entre os documentos 10 relatórios de administração e 4 entrevistas (Torres, Serra, Ferreira \& Almeida, 2013, p. 277).

Estudos baseados em poucas entrevistas, em geral, são de difícil publicação. Reay (2015) sugere um "número mágico" de 30 entrevistas. Ainda assim, as entrevistas não podem ser curtas. O volume de dados tem que ser suficiente. Isto também vale para os documentos. Por exemplo, Ribeiro (2016) num estudo em curso sobre tomada 
de decisão estratégica em um Conselho de Administração de uma empresa familiar, utilizou 38 atas de reuniões do conselho, em um total de 443 páginas de documentos, juntamente com 58 páginas transcritas de duas gravações de reuniões. Tanto Myers (2009), que apresenta sugestões para coleta de dados, como Patton (2002) sobre dados de entrevistas, concordam que a amplitude e a profundidade dos dados são cruciais. Salvato e Corbetta (2013) é usado, por nós, como um exemplo de um estudo qualitativo sem estruturado que se usa de entrevistas para a coleta de dados. Neste estudo os autores utilizaram 12 entrevistas e apresentam uma argumentação sólida e profunda na seção de método. Ou seja, é importante que os artigos qualitativos expliquem e demostrem a quantidade e qualidade dos dados (Chenail, 2009).

- Estratégia \#2 - Estabelecer uma questão de pesquisa apropriada para guiar o artigo

Todos os artigos acadêmicos necessitam ter uma questão de pesquisa que guia o artigo (Ferreira 2015). O ponto de partida é essa questão de pesquisa que, mesmo podendo ser relativamente abrangente, é suficientemente clara e precisa (Gioia, Corley \& Hamilton, 2012). De salientar que a questão de pesquisa, mesmo que formulada $a$ priori pode, e possivelmente deve, ir sendo ajustada com o decurso da maturação do projeto e na realização e redação do estudo. A questão de pesquisa remete para a lacuna a ser estudada e será um ponto de foco dos revisores do artigo que vão procurar encontra-la já na introdução do artigo (Reay, 2015). No seu editorial, Balsal e Corley (2012) sugerem o artigo de Plowman, Baker, Beck, Kulkarni, Solansky e Travis (2007, p. 516) como exemplo de especificação de uma questão de pesquisa que orienta toda a condução do artigo:

In this research, we attempt to understand how and why an initial small change, whose ultimate consequences were unintended, escalated and led to radical organizational change.

Os estudos de natureza quantitativa constroem e argumentam hipóteses (que derivam da questão de pesquisa), estabelecendo os construtos e indicando formas de mensuração, mas, no entanto, não conseguem avaliar os processos organizacionais (Gioia et al., 2012). Em contraste, as questões de pesquisa de estudos qualitativos visam responder a questões "como" e "porque". A pergunta "como" oferece a oportunidade de compreender melhor processos e situações difíceis de serem aferidas nos estudos quantitativos. No artigo de Torres et al. (2013, p. 274) comentado anteriormente, por exemplo, o objetivo reproduzido a seguir reflete a necessidade de compreender melhor como acontece o declínio organizacional:

Neste artigo, respondemos à chamada para investigar o declínio das organizações e, em especial, focamos o declínio de uma empresa brasileira de grande porte: a Gradiente, ao buscar compreender o contexto e o que levou ao seu declínio. O artigo tem como objetivo, a partir do estudo longitudinal de 35 anos da empresa, apresentar um modelo substantivo que explique $o$ declínio da Gradiente.

- Estratégia \#3 - Baseie seu estudo na literatura relevante

Em comentário editorial anterior - veja na aba "How to publish (or perish?)" no site da RIAE/IJSM - apresentamos os aspectos importantes da revisão de literatura para qualquer artigo acadêmico. Como foi mencionado anteriormente, nos artigos qualitativos, em geral, não são apresentados modelos propositivos a priori, mas na revisão de literatura se direciona para a lacuna a ser respondida, e que mostra o que já foi investigado sobre o tema, e que é especificamente relevante para o que está sendo estudado (Ferreira, 2015).

- Estratégia \#4 - Explique seus métodos e mostre o seu trabalho

A clareza é um elemento crucial em todos os tipos de estudos. Mesmo assim, talvez a apresentação do método seja ainda mais crítica nos estudos qualitativos. Isto significa a necessidade de garantir a transparência e clareza na descrição do "processo" de pesquisa (Gioia et al., 2013). Reay (2015) sugere que seja utilizada bibliografia básica, em livros dedicados à metodologia qualitativa. Em uma revisão de desk da própria Trish Reay de um artigo nosso em andamento cujo primeiro autor é Marcio Marietto, a editora comenta:

The way in which you present your methodology is a significant concern. You have explained that you conducted interviews and video-recordings, and it seems that you have rich, interesting data. However, you haven't provided enough information about your data collection or analysis strategies to convince your readers that you have 
Cuidados a Tomar nos Artigos com Pesquisa Qualitativa $\begin{array}{lrr}\text { conducted } & \text { your } & \text { work } \\ \text { systematically. } & \text { Your } & \text { only }\end{array}$ reference to qualitative methods (how to do qualitative research) is by LeBaron - and this is a keynote address. That will be helpful to you, but you should also read and reference books or articles specifically focused on qualitative methods. You must show us that you followed acceptable qualitative methods.

One of my favorite books on qualitative methodology (but there are many others!) is the following: Myer, M. 2013. Qualitative Research in Business and Management. Sage. You might find it to be helpful.

Há dois templates que são, porventura, mais utilizados para a apresentação de pesquisas qualitativas (Langley \& Abdallah (2011): o de Eisenhardt e o de Gioia. Eisenhardt é positivista e muito utilizada na análise comparativa de casos. $\mathrm{O}$ trabalho de Gioia é interpretivista e também muito utilizado em estudos qualitativos em geral. Este tipo de forma de apresentação tem sido característica dos estudos qualitativos publicados em periódicos de renome recentes, em especial no Academy of Management Journal. Exemplos deste tipo podem ser verificados no artigo de Salvato e Corbeta (2013), já mencionado, e no artigo de Corley e Gioia (2004), entre outros.

Um exemplo de apresentação de análise conforme Corley e Gioia está apresentado a seguir de um artigo em curso. A figura 1 apresenta os grupos de dados de $1^{\text {a }}$ ordem, que são organizados segundo temas de $2^{\mathrm{a}}$ ordem, que por sua vez possibilitam um maior nível de abstração pela proposição de dimensões agregadas que ajudam na compreensão da teoria emergente (Gioia, Corley \& Hamilton, 2012; Reay, 2015). Ou seja, não basta apresentar segmentos de texto para compor a apresentação dos resultados e é necessário realmente interpretar, mas segundo procedimentos claros para não parecer que tudo são opiniões.

Figura 1 - Exemplo de organização segundo framework de Gioia



Fonte: Trabalho em andamento dos autores. 
É importante apresentar os critérios e a dimensão da codificação e explicar se e como a pesquisa foi auxiliada por software analíticos. Por exemplo, Ribeiro (2016) utilizou o software MaxQda, mas há outros como o Atlas.ti e NVivo. O trabalho de Ribeiro (2016, p. 7) descreve claramente a forma como fez a codificação dos dados coletados.

A partir dos 793 segmentos codificados nas 38 Atas de reunião do Conselho e em duas reuniões gravadas e transcritas. Foram identificados 47 códigos primários que originaram oito códigos finais, sendo eles: foco operacional, foco estratégico, expansão, retrenchment, cenários desfavoráveis, conflitos, baixa sinergia dos negócios e centralização de poder. Tanto a codificação quanto a análise foram realizadas com o auxílio do Software MaxQda v. 12.1, onde efetuou-se a observação das ocorrências simultâneas dos códigos - co-ocorrências - os códigos primários e finais identificados são apresentados no Figura 1.

As técnicas de codificação podem ser verificadas em bons livros de pesquisa qualitativa.

- Estratégia \#5 - Conte uma história empírica intrigante

A elaboração de um artigo qualitativo com rigor metodológico não significa que precisa ser maçante (Salvato \& Aldrich, 2012). Uma história persuasiva é um aspecto crítico dos trabalhos qualitativos. A apresentação dos resultados em um artigo qualitativo é contar uma história empírica (Golden-Biddle \& Locke, 2006). Desta história empírica é que aparece o modelo emergente da pesquisa. Segue exemplo a partir do artigo de Torres et al. (2013). A Figura 2 apresenta as relações a partir da análise com o software Atlas.ti.

Figura 2 - Mapa de coocorrências a partir do Atlas.ti

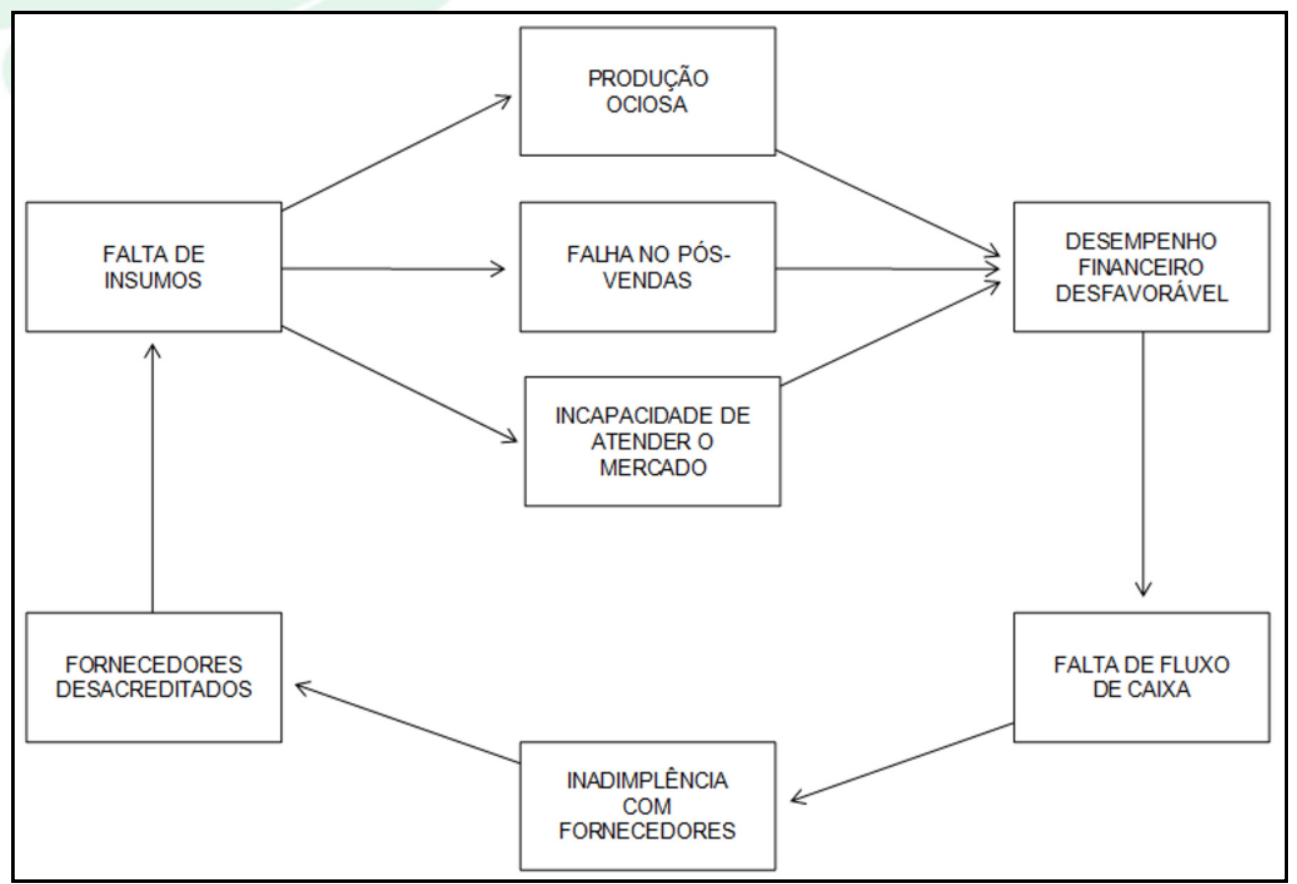

Fonte: Torres et al. (2013). 
Cuidados a Tomar nos Artigos com Pesquisa Qualitativa

A Figura 3 apresenta um exemplo de teoria substantiva, a partir do trabalho de Torres et al.
(2013), que emergiu dos dados e que passa a ser discutida a luz da teoria na seção de discussão.

Figura 3 - Exemplo de teoria emergente

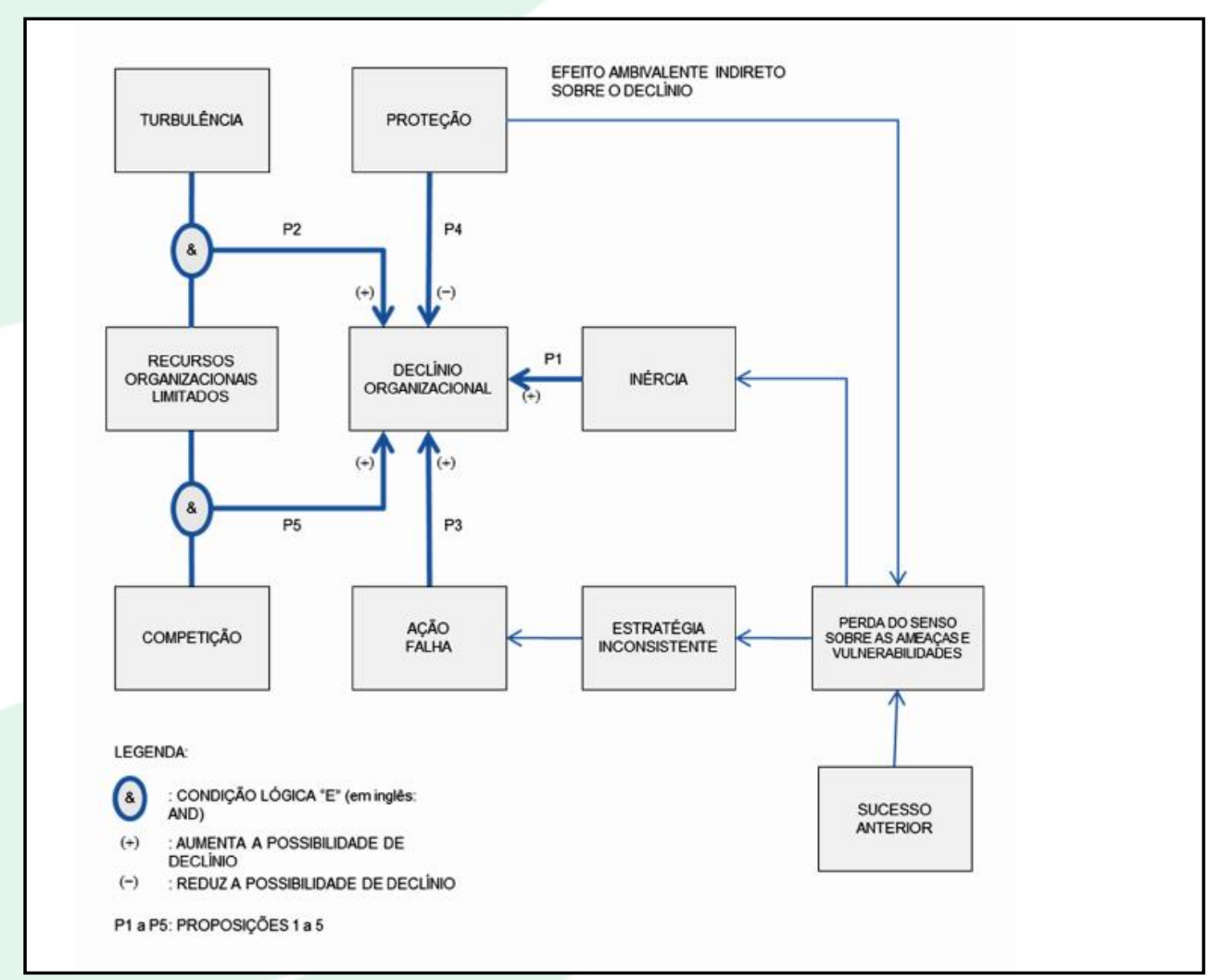

Fonte: Torres et al. (2013). 
A parte final do trabalho qualitativo pode ser mais extensa e talvez mais complexa que num artigo quantitativo. Efetivamente, mesmo os resultados podem exigir mais "espaço" porque os resultados de estudos qualitativos podem ser mais difíceis sistematizar e consolidar em tabelas. A discussão e contribuição pode ser representada em esquemas, mas precisam de texto de explicação e argumentação.

- Estratégia \#6 - Conte uma história teórica convincente

Ressaltamos a importância da seção de discussão do trabalho (ver mais detalhes em Ferreira, 2015). A história empírica, a partir da teoria emergente substantiva encaminha a discussão com a conversação a partir da literatura anterior. Esta história teórica encaminha para entregar uma contribuição. Aqui se compara os resultados com a literatura anterior, tendo o cuidado de estar conectada com a revisão de literatura do trabalho. Ou seja, a teoria é o elemento crucial. Note que é a teoria e não o caso, os participantes ou os dados, onde deve colocar o foco.

\section{- Estratégia \#7 - Apresentar uma contribuição clara}

Seguindo a estratégia \#6, a contribuição é crucial. $\mathrm{O}$ artigo precisa ter e explicar a contribuição para a teoria. A contribuição é exposta logo no início, na seção de Introdução e desenvolvida na seção final de Discussão. Todos os estudos precisam ter uma contribuição e talvez uma forma simples de entender o que isto significa seja pensar, como refere Ferreira (2015) o que os leitores vão aprender com o seu artigo.

\section{E O QUE MAIS...}

Neste comentário editorial apresentamos alguns aspectos relevantes sobre pesquisa de qualidade, com sugestões mais direcionadas para a pesquisa qualitativa. $\mathrm{O}$ intuito primordial é chamar atenção aos pesquisadores, especialmente para as suas submissões a RIAE/IJSM, de alguns elementos a contemplar para que seus estudos qualitativos tenham maior possibilidade de serem aceitos ou bem avaliados pelos nossos revisores. Para este fim, fizemos algumas comparações, por vezes talvez simplificadas, considerando estudos seguindo abordagens quantitativas e qualitativas.

Talvez entre as principais recomendações esteja a que retiramos de Kohler (2016) que enunciou que os trabalhos qualitativos precisam apresentar em maior detalhe os elementos do contexto e escolha do contexto da pesquisa, com uma descrição clara dos objetos (amostra ou participantes), procedimentos na condução da pesquisa (método utilizado e a aplicabilidade do método ao estudo), o processo de coleta de dados, e o processo de análise dos dados (por exemplo, de codificação caso exista).

Parece ser comumente aceito que os estudos qualitativos têm vantagens sobre os quantitativos quando buscamos entender processos nas organizações, por exemplo, e acessar fenômenos ainda pouco compreendidos, trazendo à luz novos conceitos e aspectos de contextos mais específicos. Ou seja, têm o potencial de gerar nova teoria e possibilitar estender a teoria já acumulada em meios que os estudos quantitativos têm maior dificuldade. Talvez possamos enunciar algumas áreas que, em estratégia os estudos qualitativos podem ter vantagens, como seja nos aspectos ligados a oportunismo, capacidades dinâmicas, tomada de decisão estratégica, estratégia como prática, estratégia de conhecimento, e vários aspectos comportamentais em geral. Mas, mesmo nestas áreas temos estudos quantitativos já publicados, pelo que a abordagem e o foco específico terão que ser distintos. O fundamental, portanto, talvez nem esteja apenas nos temas, mas no rigor dos trabalhos submetidos e, talvez, no entendimento dos métodos, técnicas e cuidados a ter que, na sua ausência, prejudicam substancialmente os artigos submetidos.

Com o intuito de ajudar os pesquisadores recomendamos algumas leituras que nos ajudaram e lhes podem ser úteis:

Bansal e Corley (2012) no editorial da AMJ, apresentam as características da pesquisa qualitativa.

Duriau, Reger e Pfarrer (2007) apresentam a utilização de análise de conteúdo em Administração.

Gioia, Corley e Hamilton (2012), apresentam os aspectos que garantem o rigor em uma pesquisa qualitativa indutiva.

Kohler (2016) em um editorial da AMLE, apresenta os aspectos importantes a considerar em artigos qualitativos, incluindo a sugestão de uma check-list.

Reay (2014) enuncia sete estratégias a considerar na publicação de artigos qualitativos.

Savall, Zardet, Bonnet \& Péron (2008), pesquisaram dezenas de anos de revisões de artigos qualitativos e apresentam dez critérios desenvolvidos indutivamente a partir do ponto de vista dos revisores.

Para concluir, temos consciência que este comentário editorial não é a solução para os 
problemas que se colocam em termos de qualidade nas pesquisas e artigos. Talvez nem seja exatamente um alerta porque a situação tem sido relatada, pelo menos em fóruns informais. Mas é um ponto de partida para promover o debate, que pode ser continuado, por exemplo, em sessões de painel nos eventos como o EnANPAD, incluindo mais disciplinas de metodologia nos programas de mestrado e doutorado, nas políticas editoriais dos periódicos, e nas relações de orientação entre $o$ professor e seus estudantes. O objetivo não é criticar o método, porque se reconhece a sua relevância, mas sim caminhar para o maior aprimoramento em pesquisa qualitativa de modo que pesquisadores brasileiros publiquem seus estudos nos melhores periódicos internacionais.

Talvez fosse relevante entender o porquê efetivamente do grande volume de estudos qualitativos que é realizado no Brasil, mesmo para situações, contextos e objetos onde pareceria que as metodologias quantitativas com testes de hipóteses seriam mais apropriadas. Talvez também entender quem está realmente a realizar estes estudos e qual a predominância de alunos de mestrado e doutorado na gênese dos estudos. Por fim, entender se alguma "tradicional" fuga a questões de estatística podem encaminhar os estudantes para estes trabalhos. Ou seja, entender o porquê, o quem e o como da pesquisa qualitativa em Administração e especificamente em estratégia no Brasil.

Uma nota final para notar que não focamos em demasia o caso específico dos estudos de caso, mas podemos relatar sobre o elevado número de estudos de casos que recebemos para avaliação e oportunamente estaremos apresentando um comentário editorial especialmente dedicado a este tipo de pesquisa predominantemente qualitativa.

\section{REFERÊNCIAS}

Aguinis, H., Pierce, C., Bosco, F., \& Muslin, I. (2009). First decade of Organizational Research Methods: Trends in design, measurement, and data-analysis topics. Organizational Research Methods, 12, 69112.

Bansal, P., \& Corley, K. (2012). Publishing in AMJ - Part 7: What is different in qualitative research. Academy of Management Journal, 55(3), 509-513.

Barbosa, J., Ponte, V., Oliveira, M., \& Moura, H. (2008). Análise das metodologias e técnicas de pesquisas adotadas nos estudos brasileiros sobre Balanced Scorecard: Um estudo dos artigos publicados no período de 1999 a 2006.
In J. Lopes, J. Francisco \& M. Pederneiras (Orgs.), Educação contábil: tópicos de ensino e pesquisa. São Paulo: Atlas.

Bettis, R., Gambardella, A., Helfat, A., \& Mitchell, W. (2015). Qualitative empirical research in strategic management. Strategic Management Journal, 36, 637-639

Chenail, R. (2009). Communicating your qualitative research better. Family Business Review, 22, 105-108.

Corley, K., \& Gioia, D. A. (2004). Identity ambiguity and change in the wake of a corporate spin-off. Administrative Science Quarterly, 49, 173-208.

Duriau, V., Reger, R., \& Pfarrer, M. (2007). A content analysis of the content analysis literature in organization studies: Research themes, data sources, and methodological refinements. Organizational Research Methods, 10(5), 5-34.

Falaster, C., Ferreira, M., \& Canela, R. (2015). Motivos de rejeição dos artigos nos periódicos de Administração. Organizações \& Sociedade, 23(77), 285-306.

Ferreira, M. (2015). Pesquisa em Administração e Ciências Sociais. Rio de Janeiro: RJ, LTC.

Fiates, G., Serra, F., \& Martins, C. (2014). A aptidão dos pesquisadores brasileiros pertencentes aos programas de pós-graduação stricto sensu em Administração para pesquisas quantitativas. Revista de Administração,49(2), 384-398.

Gibbert, M., \& Ruigrok, W. (2010). The what and how of case study rigor: Three strategies based on published work. Organizational Research Methods, 13(4), 710-737

Gioia, D., Corley, K., \& Hamilton, A. (2012). Seeking qualitative rigor in inductive research: Notes on the Gioia Methodology. Organizational Research Methods, 16(1), 1531.

Golden-Biddle, K., \& Locke, K. (2006). Composing qualitative research. Thousand Oaks, CA: Sage.

Kohler, T. (2016). On writing up qualitative research in Management Learning and Education. Academy of Management Learning \& Education, 15(3), 400-418. 
Myers, M. D. (2009). Qualitative research in business \& management. London, England: Sage.

Nascimento, A., Junqueira, E. \& Martins, G. (2010). Pesquisa acadêmica em contabilidade gerencial no Brasil: Análise e reflexões sobre teorias, metodologias e paradigmas. Revista de Administração Contemporânea, 16(6), 11131133.

Patton, M. (2002). Qualitative research and evaluation methods. Thousand Oaks, CA: Sage Publications.

Phelan, S., Ferreira, M. \& Salvador, R. (2002). The first twenty years of the Strategic Management Journal: 1980- 1999. Strategic Management Journal, 23(12), 1161-1168.

Plowman, D., Baker, L., Beck, T., Kulkarni, M., Solansky, S., \& Travis, D. (2007). Radical change accidentally: the emergence and amplification of small change. Academy of Management Journal, 50, 515-543.

Podsakoff, P., \& Dalton, D. (1987). Research methodology in organizational studies. Journal of Management, 13, 419-41.

Reay, T. (2014). Publishing qualitative research. Family Business Review, 27(2): 95-102.

Ribeiro, I. (2016). Influência da composição do Conselho na tomada de decisão estratégica em um grupo empresarial de grande porte. In Enanpad 2016.

Salvato, C., \& Aldrich, H. (2012). "That's interesting!" in family business research. Family Business Review, 25, 125-135.

Salvato, C., \& Corbetta, G. (2013). Transitional leadership of advisors as a facilitator of successors' leadership construction. Family Business Review, 26, 235-255.

Serra, F., Fiates, G. \& Ferreira, M. (2008). Publicar é difícil ou faltam competências? O desafio de pesquisar e publicar em revistas científicas na visão dos editores e revisores internacionais. Revista de Administração Mackenzie, 9(4), 32-55.

Serra, F., Ferreira, M., Pereira, M. \& Lisoni, J. (2008). Evolução da pesquisa em RBV: Um estudo dos últimos EnANPADs. Revista Brasileira de Estratégia, 1, pp. 39-56.
Torres, A., Serra, F., Almeida, M., \& Ferreira, M. (2013). O declínio de uma empresa brasileira de grande porte: $\mathrm{O}$ caso Gradiente. Revista de Administração e Contabilidade da Unisinos, 10(3), 273-292.

Whetten, D. (2009). Modeling theoretical propositions. In Huff, A. (Ed.), Designing research for publication, 217-250. Thousand Oaks, CA: Sage. 